\title{
A HOSPITAL BASED STUDY: IS THE PRESCRIPTION OF BENZODIAZEPINE CONSISTENT WITH THE GUIDELINES?
}

\author{
Thapa DK ${ }^{1}$, Lammichhane $\mathrm{N}^{2}$, Subedi $\mathrm{S}^{3}$
}

\section{ABSTRACTS}

OBJECTIVE: Benzodiazepines are one of the most frequently prescribed psychotropic drugs. They confer a therapeutic value in a wide range of conditions. They exert sedative/ hypnotic, anxiolytic, anticonvulsant, muscle relaxant and amnesic action. Nearly all of the disadvantages of benzodiazepines result from long term use leading to development of tolerance, dependence and withdrawal. This study was done to determine if the pattern of benzodiazepines prescription among the psychiatric patients is consistent with the guideline.

METHODS: This was a descriptive, hospital based cross- sectional study done in the psychiatry department at Gandaki Medical College. The consecutive 50 patients who either had a past history of treatment with or were still regularly on prescription for any of the following medication; alprazolam, chlordiazepoxide, clonazepam,diazepam, and lorazepamwere included in the study. The psychiatric diagnosis of the patients, duration and types of benzodiazepines dispensed to patients were worked up. The duration of study was 6 months (Jan- June 2013).

RESULTS: The total numbers of subjects enrolled in the study was 50 . Female constituted 28 (56\%), majority were married $45(90 \%)$ and most of the subjects $31(62 \%)$, were from the local district of Kaski. The mean age of the subjects was $41.1 \pm 15.3$ years.Among the types of benzodiazepines prescribed, clonazepam was the most frequently prescribed benzodiazepine. Dispensing of less than 30-days or 1 month supply of benzodiazepines, a practice typically recommended by practice guidelines, occurred in only $5(10 \%)$ of the users. The study showed that there was a huge variation regarding the duration of benzodiazepines use, ranging from the period of less than of 1 month to the maximum duration of 192 months or 16 years. The mean duration of the benzodiazepine use was $34.8 \pm 50$ months i.e. near about 3 years.

CONCLUSIONS: Despite guideline cautions, long-term benzodiazepines use remains a common treatment pattern.

KEY WORDS: Clonazepam, Duration of use, Guidelines

1. Assistant Professor, Department of Psychiatry,Gandaki Medical College \& Teaching Hospital, Kaski, Pokhara, Nepal

2. Associate Professor, Department of Psychiatry,Gandaki Medical College \& Teaching Hospital, Kaski, Pokhara, Nepal

3. Assistant Professor, Department of Psychiatry, Universal College of Medical Sciences \& Teaching Hospital, Bhairahawa, Nepal

For Correspondence

Dr. Dev Kumar Thapa

Assistant Professor,

Department of Psychiatry,

Gandaki Medical College \& Teaching ,

Hospital, Kaskipokhara, Pokhara

E-mail: ddthapa@hotmail.com 


\section{INTRODUCTION}

Benzodiazepines ${ }^{1}$ and antidepressants are the most frequently prescribed psychotropic drugs. ${ }^{2}$ Anxiolytics, hypnotics, and antidepressants each accounts for approximately $20 \%$ of all prescriptions. ${ }^{3}$ Benzodiazepines have a common effect on the receptors that have been called as benzodiazepine receptor which modulates $\square$-aminobutyric acid (GABA) activity. ${ }^{4,5}$ The enhanced effect of (GABA) results in sedative/ hypnotic (sleep-inducing), anxiolytic (anti-anxiety), anticonvulsant, muscle relaxant and amnesic action. ${ }^{4}$ Thus, confering a therapeutic value in a wide range of conditions. All benzodiazepines exert, in slightly varying degrees posses these 5 major actions. ${ }^{6}$ These properties make benzodiazepines useful in treating anxiety, insomnia, agitation, seizures, muscle spasms, motor disorders, alcohol withdrawal and as a premedicative agents. ${ }^{4}$ Few, if any, other drugs can compete with them in all these respects. ${ }^{6}$

acid (GABA) activity. ${ }^{4,5}$ The enhanced effect of (GABA) results in sedative/ hypnotic (sleep-inducing), anxiolytic (antianxiety), anticonvulsant, muscle relaxant and amnesic action. ${ }^{4}$ Thus, confering a therapeutic value in a wide range of conditions. All benzodiazepines exert, in slightly varying degrees posses these 5 major actions. ${ }^{6}$ These properties make benzodiazepines useful in treating anxiety, insomnia, agitation, seizures, muscle spasms, motor disorders, alcohol withdrawal and as a premedicative agents. ${ }^{4} \mathrm{Few}$, if any, other drugs can compete with them in all these respects. ${ }^{6}$

In short term use, benzodiazepines (BZDs) can be valuable and sometimes lifesaving. The major clinical advantages of benzodiazepines are high efficacy, rapid onset of action and low toxicity. Nearly all of the disadvantages of benzodiazepines result from long term use leading to development of tolerance, dependence and withdrawal. These have earned them a poor reputation, particularly as drugs of dependence. ${ }^{6}$ It is difficult to know whether these drugs are being inappropriately prescribed. Excessive growth in prescriptions for benzodiazepines had led to concern over their use. ${ }^{2}$ The yearly prescription for benzodiazepine anxiolytic in the UK alone was 18 million in $1978 .^{6}$ The concern was further reinforced by evidence that they were being prescribed for excessively long periods. Subsequent studies have shown that about two fifths of regular users of benzodiazepines develop pharmacological dependence and have withdrawal symptoms when the dosage of their drugs is reduced or treatment is stopped. $^{2}$

Anthierens and colleagues remind us how widespread the use of BZDs has become: in Belgium, 1 patient in 3 takes BZDs daily and in a habitual way; in Europe, a survey revealed that almost $10 \%$ of the population are taking BZDs over long periods; and in Canada, the rate of BZD use over long periods is about $3.4 \%{ }^{7}$

So benzodiazepines, since they induce dependence and abuse, most countries have formulated guidelines regarding usage of these drugs. ${ }^{8}$ Eg: NICE guidelines in UK, FDA in USA, Ministry of Health Singapore: Clinical Practice guidelines, Hong Kong College of Psychiatrists: Guidelines on the use of Benzodiazepines etc.

This study was undertaken at Psychiatry department of Gandaki Medical College, Pokhara, to determine if the pattern of benzodiazepines prescription among the psychiatric patients is consistent with the guideline.

\section{METHODS}

This was a descriptive, hospital based cross- sectional study done in the psychiatry department at Gandaki Medical College, one of the tertiary care centre in the western Nepal. The consecutive 50 patients who either had a past history of treatment with or were still regularly on prescription for any of the following medication; alprazolam, chlordiazepoxide, clonazepam,diazepam and lorazepam were included in the study. The consecutive 50 patients of either sex and of all age groups were included in the study. The patients' naïve to any of the above drug and those unwilling to participate in the study were excluded. The psychiatric diagnosis of the patients, duration and types of benzodiazepines dispensed to patients were worked up. The duration of study was 6 months, from January to June, 2013.

\section{RESULTS}

The total numbers of subjects enrolled in the study was 50 . The female constituted of $28(56 \%)$ and male were $22(44 \%)$. The majority of them i.e. $45(90 \%)$ were married, $4(8 \%)$ single and $1(2 \%)$ was widow. The age of the subjects ranged from 12 years to 80 years while the mean age was $41.1 \pm 15.3$ years. Only $12(24 \%)$ were educated up to SLC (Board examination after standard 10) or above.

Majority of the subjects $31(62 \%)$ were from the local district of Kaski. Among the rest $6(12 \%)$ were from Syanjya, $5(10 \%)$ from Parbat, 4(8\%) from Tanahun, $3(6 \%)$ from Baglung and 1 (2\%) from Myagdi.

Among the types of benzodiazepines prescribed; clonazepam was the most frequently prescribed benzodiazepine constituting of $23(46 \%)$ prescription, followed by lorazepam 13 (26\%), alprazolam $7(14 \%)$, diazepam $4(8 \%)$ and chlordiazepoxide $3(6 \%)$ prescription. (Table 1)

Table1: Showing the various benzodiazepine prescriptions

\begin{tabular}{|l|c|c|}
\hline Benzodiazepine: Types & Frequency (n) & Percentage (\%) \\
\hline Clonazepam & 23 & $46 \%$ \\
\hline Lorazepam & 13 & $26 \%$ \\
\hline Alprazolam & 7 & $14 \%$ \\
\hline Diazepam & 4 & $8 \%$ \\
\hline Chlordiazepoxide & 3 & $6 \%$ \\
\hline TOTAL & $\mathrm{n}=50$ & $100 \%$ \\
\hline
\end{tabular}

The study showed that there was a huge variation regarding the 
duration of benzodiazepines use, ranging from the period of less than of 1 to the maximum duration of 192 months or 16 years. The mean duration of the benzodiazepine use was 34.8 \pm 50 months i.e. near about 3 years.

Dispensing of less thana 30-days or 1 month supply of benzodiazepines, a practice typically recommended by practice guidelines, occurred in only $5(10 \%)$ of the users.

The clinical conditions for which the above benzodiazepines were used were many. For the F40- F48 category i.e. Neurotic, stress- related and somatoform disorders, there were altogether 21 cases comprising of $42 \%$. Among them, there were 9 cases of anxiety disorder unspecified, 6 cases of generalized anxiety disorder, 5 cases of agoraphobia with panic disorder and 1 case of persistent somatoform pain disorder.

For the F30- F39 category, i.e. mood (affective) disorders, there were altogether 8 cases comprising of $16 \%$. Among them, there were 3 cases of bipolar affective disorder, 2 cases of depressive episode, 2 cases of recurrent depressive episode and 1 case of mania with psychotic symptoms. There were altogether 3 cases of schizophrenia (F20), comprising of $6 \%$.

Table 2: Showing the distribution of clinical conditions in subjects on benzodiazepines use

\begin{tabular}{|c|c|c|c|}
\hline $\begin{array}{l}\text { Category of } \\
\text { clinical conditions }\end{array}$ & Types of disorder & $\begin{array}{l}\text { Frequency } \\
\text { (n) }\end{array}$ & $\begin{array}{c}\text { Percentage } \\
(\%)\end{array}$ \\
\hline \multirow{5}{*}{$\begin{array}{l}\text { Neurotic, stress } \\
\text { related and somatoform } \\
\text { disorders (F40-F48) }\end{array}$} & Anxiety disorder unspecified & 9 & \\
\hline & Generalized anxiety disorder & 6 & \\
\hline & $\begin{array}{c}\text { Agoraphobia with panic } \\
\text { disorder }\end{array}$ & 5 & \\
\hline & $\begin{array}{c}\text { Persistent somatoform pain } \\
\text { disorder }\end{array}$ & 1 & \\
\hline & Total & 21 & $42 \%$ \\
\hline \multirow{5}{*}{$\begin{array}{l}\text { Mood (affective) disorder } \\
\text { (F30 - F39) }\end{array}$} & Bipolar affective disorder & 3 & \\
\hline & Recurrent depressive disorder & 2 & \\
\hline & Depressive episode & 2 & \\
\hline & $\begin{array}{l}\text { Mania with psychotic } \\
\text { symptoms }\end{array}$ & 1 & \\
\hline & Total & 8 & $16 \%$ \\
\hline $\mathrm{F} 20$ & Schizophrenia & 3 & $6 \%$ \\
\hline Headache cases & Migraine Headache & 5 & $10 \%$ \\
\hline Comorbid cases & - & 11 & $22 \%$ \\
\hline \multirow[t]{3}{*}{ Others } & Chorea & 1 & $2 \%$ \\
\hline & Peripheral neuropathy & 1 & $2 \%$ \\
\hline & Overall TOTAL & $\mathrm{n}=50$ & $100 \%$ \\
\hline
\end{tabular}

There were altogether $11(22 \%)$ cases of comorbid conditions (occurrences of 2 or more conditions) from the pool of like alcohol dependence syndrome, nicotine dependence syndrome, anxiety unspecified, depressive episode, and persistent somatoform pain disorder and migraine headache. Other conditions included $5(10 \%)$ cases of migraine headache and a case each of chorea and peripheral neuropathy.

\section{DISCUSSIONS}

There is no doubt that benzodiazepines confer a therapeutic value in a wide range of conditions. "Since its advent, benzodiazepines have largely replaced barbiturate and nonbarbiturate anxiolytics and sedative/hypnotics as they are as effective and safer.'

In our study, we could see that they are being prescribed for various conditions like neurotic, stress- related and somatoform disorders (F40- 48 category), mood (affective) disorders (F30-F39), schizophrenia (F20) and various other comorbid conditions.

Among the types of benzodiazepines prescribed; clonazepam was the most frequently prescribed benzodiazepine constituting of 23 (46\%) prescription,followed by lorazepam 13 (26\%), alprazolam 7 (14\%), diazepam $4(8 \%)$ and chlordiazepoxide $3(6 \%)$ prescription. This finding was almost similar to the study by Valenstein et al, where clonazepam was the most frequently prescribed benzodiazepine, followed by lorazepam and diazepam. ${ }^{10}$

The study showed that there was a huge variation regarding the duration of benzodiazepines use, ranging from the period of less than 1 month to the maximum duration of 192 months (16 years). The mean duration of the benzodiazepine use was $34.8 \pm 50$ months i.e. near about 3 years. Dispensing of less than a 30-days or 1 month supply of benzodiazepines, a practice typically recommendedby practice guidelines, occurred in only $5(10 \%)$ of the users.

The UK Committee on Safety of Medicines advised that benzodiazepines are indicated for the short term relief (2-4 weeks only) of anxiety. Generally they are to be used in conjunction with other measures (psychological treatments, antidepressants, other drugs) as a short term cover, allowing time for more specific treatments to take effect. ${ }^{6,11,12}$ But our study showed the otherwise. In our study, the mean duration of benzodiazepines use in anxiety related disorder (F40- 48 category), was 45.19 months. Though this is very high over the ceiling there are debate for long term use of benzodiazepine in generalized anxiety disorder. Long term treatment over months or years has been much used to treat chronic generalised anxiety disorder. However, it has been found that most patients with chronic anxiety remained symptom-free for at least several months after a course of diazepam lasting 22 weeks, and in $37 \%$ there was no recrudescence of anxiety within a year. Similar sustained improvement was noted after a shorter 6-week course of 
diazepam. It is concluded that intermittent rather than long term benzodiazepine therapy is preferable in most cases. However, there remains a small core of patients who fail to benefit from psychological and other therapies and, for these patients, prolonged treatment, combined with general support, may be the only practical option. ${ }^{6}$ Therefore, long-term prescription of benzodiazepines may be considered desirable in certain circumstances when other alternatives are considered less appropriate than benzodiazepines. This may be in conditions such as chronic treatment-resistant anxiety or in patients who have confirmed dependency and are unable to withdraw successfully. ${ }^{12} \mathrm{Can}$ it not be argued for any kinds of chronic and resistant anxiety disorder? In our study the mean duration of benzodiazepine use for generalized anxiety disorder alone was of 41 months.

In depression, similarly, benzodiazepines may be added (for less than 4 weeks) to an antidepressant in the initial phase of treatment. 11 Since antidepressants' beneficial effects often do not occur for several weeks, benzodiazepines may provide immediate relief. ${ }^{10}$ Having said that, we could still see benzodiazepine used for longer period. In Valenstein et al study that included the sample of 128,029 veterans, most users (78\%) received 90 days' supply of benzodiazepines, and $61 \%$ received 180 days' supply. It further concluded that depressed patients treated in mental health settings commonly receive long-term treatment with benzodiazepines in combination with antidepressants, a pattern of use that is inconsistent with guideline recommendations..$^{10}$ Similarly, in our study, mean duration of benzodiazepine use for spectrum of depressive illness was 55 months. The note of caution is that our study had too little a sample of depressive illness. Fewer studies have examined the effectiveness of combination treatment with both antidepressants and benzodiazepines, comparedto antidepressant treatment alone. However, a meta-analysis completed by Furukawa and colleagues reported some benefits from combination treatment. They found that patients who received combination treatment dropped out of treatment less often than patients who received antidepressants alone. Patients who received combination treatment also experienced greater improvementsin depressive symptoms at 1 and 4 weeks. $^{10}$

These agents are used in mania and psychotic illness as well. Benzodiazepines are used as adjunctive treatment in the initial management of acute mania where sedation is a priority. Again, they should be used intermittently for 2 to 4 weeks till more appropriate long-term treatments are instituted. ${ }^{12}$ Benzodiazepines should not be used as monotherapy in the treatment of schizophrenia, schizophrenia-like psychoses and acute psychotic behaviour. A short-term trial of adjunctive benzodiazepines may be considered only in psychotic patients with persistent and clinically significant symptoms of anxiety, dangerous or assaultive behaviour. ${ }^{11}$ The another aspect is that in situations where anxiety is complicated by other illnesses such as schizophrenia, the risk of dependence may be considered acceptable because of the severity of the other disorder. ${ }^{12}$ In our study we found that benzodiazepines are being used for longer than duration stated by guidelines.

Yearly prescriptions for benzodiazepine anxiolytics have decreased in the UK from a peak of 18 million in 1978 to less than 10 million at present. ${ }^{6}$ Although the use of benzodiazepines has been declining, manyindividuals in the United States and other countries, particularlyelderly patients, continue to use these agents.Studies conducted between 1989 and 1997 in a variety of western countries reported that $10 \% 17 \%$ ofelderly community residents use benzodiazepines. In many studies, a high proportion of benzodiazepine recipients used these medications for long-term treatment, defined as 60 days of use. ${ }^{10}$ Despite decades of relevant basic and clinical research, active debate continues about the appropriate extent and duration of benzodiazepine use in the treatment of anxiety and related disorders. ${ }^{13}$ According to International study of expert judgment on therapeutic use of benzodiazepines, most experts favored antidepressants, usually tricyclic, for agoraphobia, panic and OCD; betablockers for specific social phobia; and benzodiazepines for GAD and adjustment disorder. Some experts favored polypharmacy, usually an antidepressant and a benzodiazepine. The majority usually advocated pharmacotherapy for 6 months or more. They recommended the same duration of treatment with benzodiazepines and other medications, except for GAD. ${ }^{14}$

The responses of the Expert Panel imply that; (1) most anxiety disorders are serious and merit vigorous, prolonged pharmacotherapy; and (2) antidepressants and benzodiazepines are effective and safe for long-term treatment of these conditions. This outcome contrasts with the widespread apprehension about long-term pharmacotherapy, especially with benzodiazepines, and some regulatory views. ${ }^{14}$ Among the 948 family physicians surveyed, almost half (46\%) did not see any problem with prescribing benzodiazepines; approximately one-quarter considered habitual use of BZDs to be justified if patients felt better and were not experiencing secondary effects; and $71 \%$ believed that it was correct to prescribe BZDs for a week. It was the older physicians who mostly considered the use of BZDs as being justified. ${ }^{7}$ This is further to add that the doctors' attitudes generally conflict with practice guidelines.

Thus the use of benzodiazepine anxiolytics and hypnotics continues to excite controversy. Views differ from expert to expert and from country to country as to the extent of the problem, or even whether long-term benzodiazepine use actually constitutes a problem. ${ }^{15}$ 
This small hospital based study showed that the benzodiazepines use is inconsistent with guidelines. Drawback of the study is that such a small sample is unlikely to reflect the method of benzodiazepines prescription among the masses. Current study lacks data regarding their use for insomnia, alcohol use disorder and variety of psychosis.

The riddle that needs to be solved is why is there such inconsistent use? The reasons could be many.First, it is likely that many patients find benzodiazepines helpful and request these medications from their physicians. ${ }^{10}$ Having experienced good initial symptom relief with benzodiazepine treatment, patients may also be reluctant to taper it down. ${ }^{16}$ In qualitative studies, general medical physicians reported that they prescribe long-term benzodiazepines because they are continuing prescriptions initiated by other physicians or are responding to patients' requests and avoiding disruptions in the relationship with the patient. Physicians may also begin treatment with benzodiazepines for early symptom relief and then fail to reassess the patient's need forthe medication or may feel reluctant to discontinue the medicationif the patient is doing better. ${ }^{10}$ In country like ours where we lack the necessary psychological intervention, it could be a likely reason for the prescribers to give greater emphasis to medication use. Finally, often patients don't regularly come for the follow- up assessment. Once they feel better with the initial treatment they are likely to continue with the initial regime of treatment.

\section{CONCLUSION}

A practice typically recommended by practice guidelines, occurred in only $5(10 \%)$ of the users only. Despite guideline cautions, long-term benzodiazepines use remains a common treatment pattern.

\section{REFERENCES}

1. Gorwood P, Lejoyeux M. Proper and improper use of benzodiazepines. Rev Prat. 1994 Nov 1; 44(17):2320-3. PMid:7984940

2. TyrerP.Prescribing psychotropic drugs in general practice. $\mathrm{Br}$ Med J (Clin Res Ed). 1988 Feb 27;296(6622):588-9. http://dx.doi.org/10.1136/bmj.296.6622.588

3. Linden $M$, Lecrubier $Y$, Bellantuono $C$, Benkert $O$, Kisely $S$, Simon $G$. The prescribing of psychotropic drugs by primary care physicians: An international collaborative study. $J$ ClinPsychopharmacol. 1999 Apr; 19(2):132-40. http://dx.doi.org/10.1097/00004714-199904000-00007 PMid:10211914

4. Page $C$, Michael $C$, Sutter $M$, Walker $M$, Hoffman $B B$. Integrated Pharmacology. 2nd ed. C.V.Mosb; 2002
5. Sadock BJ, VA Sadock VA, Sussman N. Kaplan and Sadock's pocket handbook of psychiatric drug treatment. 4th ed. Philadelphia: Lippincott Williams \& Wilkins;2006.

6. Ashton H. Guidelines for the rational use of benzodiazepines. When and what to use. Drugs. $1994 \mathrm{Jul} ; 48(1): 25-40$. http://dx.doi.org/10.2165/00003495-199448010-00004 PMid:7525193

7. Ladouceur R. Benzodiazepines: good or bad medicine? Can Fam Physician. 2010 Nov;56(11):1097, 1099.

8. Guidelines for the use of Benzodiazepines in office practice in the state of Maine; www.benzos.une.edu/documents /prescribingguidelines3-26-08.pdf(Date: 14/11/2013)

9. Busto UE, Sellers EM. Anxiolytics and sedative/hypnotics dependence. BrJAddict. 1991 Dec;86(12):1647-52. http://dx.doi.org/10.1111/j.1360-0443.1991.tb01760.x PMid:1686199

10. Valenstein M1, Taylor KK, Austin K, Kales HC, McCarthy JF, Blow FC. Benzodiazepine use among depressed patients treated in mental health settings. Am J Psychiatry. 2004 Apr;161(4):654-61.

http://dx.doi.org/10.1176/appi.ajp.161.4.654 PMid:15056511

11. Prescribing of Benzodiazepines, Singapore MOH Clinical Practice Guidelines 2/2008

12. Lorna Marie West. Benzodiazepines: Benefits versus risks Journal of the Malta College of Pharmacy Practice Issue 13 Summer 2007, pp 24-26

13. Uhlenhuth $E H$, Balter MB, Ban TA, Yang $K$. International study of expert judgment on therapeutic use of benzodiazepines and other psychotherapeutic medications: $I V$. Therapeutic dose dependence and abuse liability of benzodiazepines in the longterm treatment of anxiety disorders. J ClinPsychopharmacol. 1999 Dec; 19(6 Suppl 2):23S-29S.

http://dx.doi.org/10.1097/00004714-199912002-00005 PMid:10587281

14. Uhlenhuth EH, Balter MB, Ban TA, Yang K. International study of expert judgement on therapeutic use of benzodiazepines and other psychotherapeutic medications: II. Pharmacotherapy of anxiety disorders. JAffect Disord. 1995 Dec 18;35(4):153-62. http://dx.doi.org/10.1016/0165-0327(95)00064-X

15. Lader M, Tylee A, Donoghue J. Withdrawing benzodiazepines in primary care. CNSDrugs. 2009; 23(1):19-34. http://dx.doi.org/10.2165/0023210-200923010-00002 PMid:19062773

16. Cloos JM, Ferreira V. Current use of benzodiazepines in anxiety disorders. CurrOpin Psychiatry. 2009 Jan;22 (1):90-5. http://dx.doi.org/10.1097/YCO.0b013e32831a473d PMid: 19122540 